\title{
Immunohistochemical Changes after Metoclopramide Administration in Rat Brain Cells ${ }^{\dagger}$
}

\author{
Seren Gülşen Gürgen 1,*, Ayşe Tuç Yücel ${ }^{1}$, Nurcan Umur ${ }^{1}$ and Gülce Naz Yazıcı ${ }^{2}$ \\ 1 School of Vocational Health Service, Manisa Celal Bayar University, Manisa 45140, Turkey; \\ aysetuc@gmail.com (A.T.Y.); nurcanumur@gmail.com (N.U.) \\ 2 Faculty of Medicine, Erzincan University, Erzincan 24100, Turkey; gulcenazyazici.ank@gmail.com \\ * Correspondence: serengurgen@yahoo.com \\ + Presented at the 2nd International Cell Death Research Congress, Izmir, Turkey, 1-4 November 2018. \\ Published: 6 December 2018
}

\begin{abstract}
Metoclopramide, used as an anti-emetic drug in clinical practice, has recently also begun being used to establish hyperprolactinemic effects in the breastfeeding. The purpose of this study was to investigate the potential side-effects of metoclopramide applied in the lactation period in the central nervous system of infant rats. 18 female albino Wistar rats that had just given birth were divided into 3 groups together with their pups: Healthy controls, low-dose metoclopramide (10 $\mathrm{mg} / \mathrm{kg}$, twice per day i.p.) and a high-dose metoclopramide group ( $45 \mathrm{mg} / \mathrm{kg}$, twice per day i.p.). Brain tissues from 6 pups from each mother were harvested at the end of the 21st day. Immunohistochemical technique was performed using dopamine D2 receptor (DRD2), brain derived neurotrophic factor (BDNF) and neural growth factor (NGF), markers of extrapyramidal reaction in the brain, as signal molecules. Based on immunohistochemical results, DRD2 expression decreased only in the external pyramidal layer neurons in the high-dose infant group. Strong BDNF reaction was determined in pyramidal neurons in all layers in the control infant group, and decreased reaction was observed in the high- and low-dose groups. No significant difference was observed in NGF expression between the three groups. Since high-dose metoclopramide caused a decrease in DRD2 expression in the external pyramidal layer in the prefrontal cortex, and since both high and low doses reduced BDNF expression, care needs to be taken with the use of metoclopramide in the lactation period due to the possibility of extrapyramidal reactions in infants.
\end{abstract}

Keywords: brain; extrapyramidal reactions; metoclopramide

\section{Introduction}

Metoclopramide (Metpamid), sulpiride (Zegerid), domperidone (Motilium), chlorpromazine Metoclopramide easily passes the blood-brain barrier and can cause side-effects such as extrapyramidal system movement disorders (extrapyramidal reactions) [1]. Metoclopramide is used to increase lactation by breastfeeding mothers and is known to pass into milk. A milk/plasma ratio of 1.8-1.9 has been reported [2]. Metoclopramide has been observed to lead to fatigue, headache, anxiety, intestinal disorders and particularly depression and extrapyramidal side-effects $(1 \%)$ in mothers [3]. In contrast to the side-effects in the mother, whether it causes side-effects in the newborn is still the subject of debate. According to some authors, no side-effects are observed, while others regard metoclopramide passing into milk as a risk [4]. The purpose of this study was to investigate distribution of expression of dopamine receptor D2 (DRD2), an extrapyramidal reaction marker, brain-derived neurotrophic factor (BDNF) and neural growth factor (NGF) in the cerebral cortex and serum of newborn pups of mother rats administered metoclopramide during the lactation period. 


\section{Materials and Methods}

Young female Wistar Albino rats that had just given birth were used. All animal experiments were carried out in accordance with the European Communities Council Directive of 24 November 1986 (86/609/EEC) and were approved by the Animal Care Committee of Celal Bayar University.

The study began with 18 young female Wistar-albino rats and their 1-day-old pups. Mother rats in the lactation period were administrated metoclopramide (Sigma-Aldrich, St. Louis, MO, USA) for hyperprolactinemia. Mother rats and their pups were divided into 3 experimental groups containing 6 rats each. Experimental groups consisted of a control group, a low-dose metoclopramide group (10 $\mathrm{mg} / \mathrm{kg}$, 21 days, 2 doses i.p. per day) and a high-dose metoclopramide group ( $45 \mathrm{mg} / \mathrm{kg}$, 21 days, 2 doses i.p. per day). All infants were sacrificed at 10:00 a.m. at the end of the 21st day. Brain tissues were rapidly excised and placed in $10 \%$ neutral formalin for histological investigation.

Immunohistochemical Methods: At the end of the experiment, pups' brain prefrontal cortex tissues were harvested. Tissue samples processed for embedding in paraffin wax according to routine protocols. Paraffin blocks of the tissue were cut in $5 \mu \mathrm{m}$ sections and prepared for immunohistochemical staining. The slides were stained with primary antibodies DRD2 (Mouse monoclonal, Santa Cruz, CA, USA), BDNF (Rabbit polyclonal antibody, Santa Cruz, CA, USA) and NGF (Mouse monoclonal antibody, Abcam, Cambridge, MA, USA) using immunohistochemical methods. The slides were analyzed under an Olympus (CX41, Tokyo, Japan) light microscope. Two observers blinded to the experimental information evaluated the immunolabeling scores independently. The staining intensity of the slides with their immunohistochemical protocol was graded semi-quantitatively, and the HSCORE was calculated using the following equation: HSCORE $=\Sigma \mathrm{Pi}(\mathrm{i}+1)$, where $\mathrm{i}$ is the intensity of staining with a value of 1,2 or 3 (weak, moderate or strong, respectively), and where $\mathrm{Pi}$ is the percentage of stained cells for each intensity, varying from 0 to $100 \%$. The study groups were analyzed using Kruskal-Wallis nonparametric tests. All statistical analyses were performed using SPSS version 15.0 for Windows software.

\section{Results}

Infant Brain Tissue DRD2, NGF and BDNF Immunochemistry Findings Examination of the infant prefrontal cortex region revealed no DRD2 expression in the molecular layer in the control group. However, strong cytoplasmic and granular form expression was observed in the pyramidal neurons in the external pyramidal layer $(p<0.05)$ (Figure 1(A1,A2)). Similarly, quite powerful cytoplasmic and granular form immunoreaction was observed in pyramidal neurons in the internal pyramidal layer $(p<0.05)$ (Figure 1(A3)). In the low-dose metoclopramide group, expression was weak in the molecular and external pyramidal layer of the prefrontal cortex, while moderately and granular form reaction was noted in some neurons in the internal pyramidal layer (Figure 1(B1,B2,B3)). In the high-dose group, quite strong granular form DRD2 expression was determined in the internal pyramidal cell layer $(p<0.05)$, but less than that of the control group in the external pyramidal layer of the prefrontal cortex (Figure 1(C1,C2,C3)). BDNF expression in the prefrontal cortex was quite strong in neurons in the external pyramidal layer in the control group, and moderate in the other two groups $(p<0.05)$. (Figure not showen). Expression ranging from weak to moderate was observed in NGF in all groups. There was no significant difference between the groups $(p>0.05)$. (Figure not showen) (Table 1). 

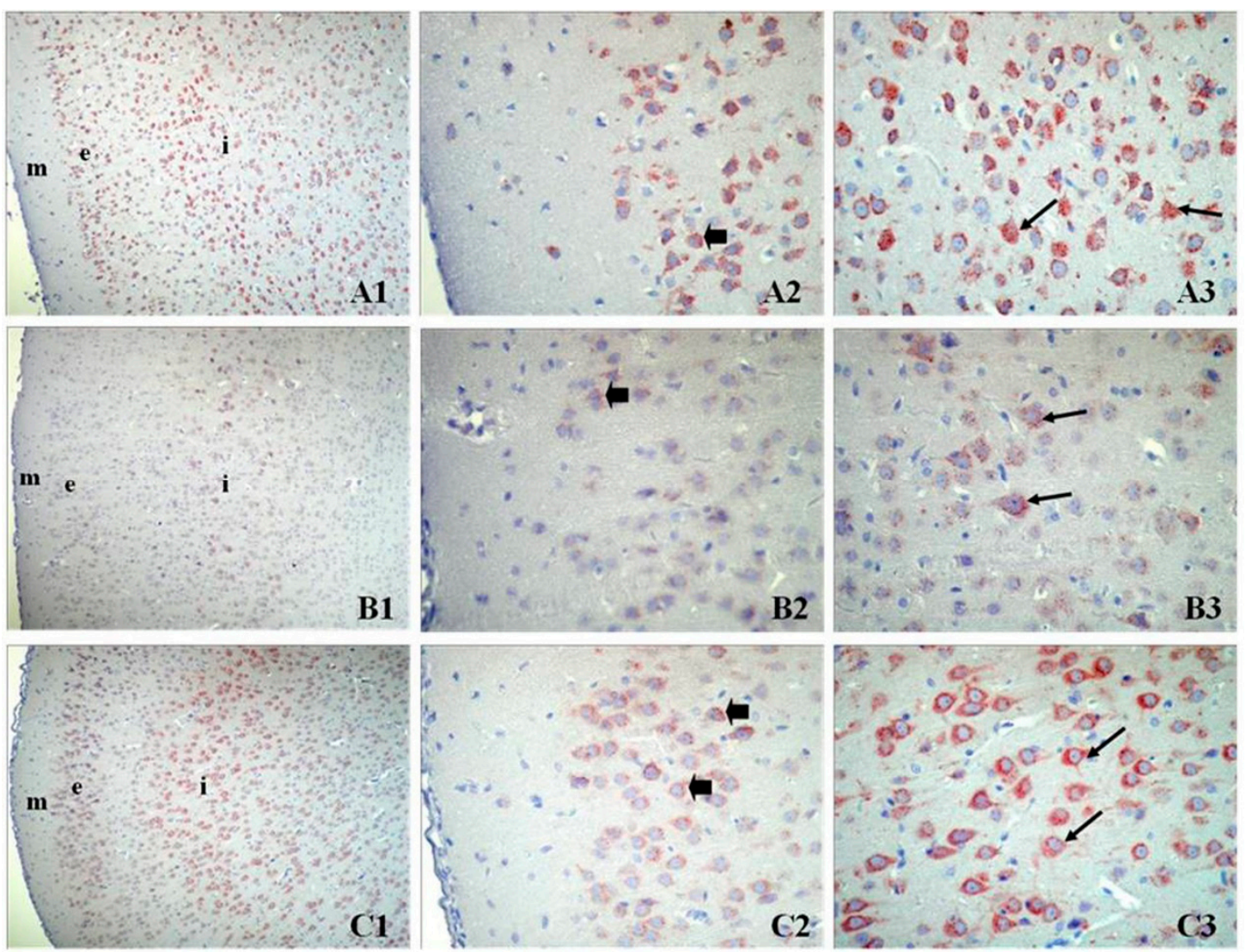

Figure 1. Prefrontal cortex DRD2 immunostaining. Control (A), low-dose metoclopramide (B), highdose metoclopramide (C). m: molecular layer, e: external pyramidal layer, i: internal pyramidal layer $(1) \times 100, \Rightarrow$ : external pyramidal neurons, $\rightarrow$ : internal pyramidal neurons, external pyramidal layer (2), internal pyramidal layer (3). $\times 400$.

Table 1. DRD2, BDNF, NGF H-score levels in the brain prefrontal cortex of infant rats in the control and low- and high-dose metoclopramide groups.

\begin{tabular}{ccccc}
\hline \multirow{2}{*}{ H-Score } & \multicolumn{2}{c}{ DRD2 } & BDNF & NGF \\
\cline { 2 - 5 } & EP DRD2 & IP DRD2 & EP BDNF & EP NGF \\
\hline Control & $227.3 \pm 21.4$ & $235.3 \pm 10.6$ & $297.3 \pm 31.1$ & $176.7 \pm 7$ \\
Low-dose & $121 \pm 3.8$ & $150.7 \pm 5.2$ & $168.6 \pm 8.6$ & $123 \pm 19.3$ \\
High-dose & $131.3 \pm 5.2$ & $218 \pm 7.5$ & $164.6 \pm 12.1$ & $118.5 \pm 5.6$ \\
\hline
\end{tabular}

EP: External pyramidal layer, IP: Internal pyramidal layer, DRD2: Dopamine D2 receptor, BDNF: Brain derived neurotrophic factor, NGF: Neural growth factor * $p<0.05$ one-way analysis of variance ANOVA, data are expressed as mean \pm SD.

\section{Discussion}

Metoclopramide is known to pass into milk [2]. Some authors describe metoclopramide as an "inverse neuroleptic" [5]. Some authors classify metoclopramide as a neuroleptic but as an antipsychotic agent with a weak effect [6]. In this study DRD2 decreasing in both internal and external pyramidal neurons in the low-dose metoclopramide group and in the external pyramidal neurons in particular in the high-dose group shows that this derives from metoclopramide's dopamine D2 receptor antagonistic effect [7]. BDNF, which shares a high degree of structural homology with NGF, is a member of the neurotropin family of molecules, such as neurotropin-3 (NT-3) and neurotropin4 (NT-4). NGF has been shown to reduce neuron degeneration in rats and to increase peripheral nerve regeneration [6]. Antipsychotic drugs are also known to alter neurotropin levels in the brain and to cause various side-effects (such as extrapyramidal and anticholinergic symptoms, tardive dyskinesia and hyperprolactinemia). Additionally, recent studies using animal models of schizophrenia have 
shown that NGF and BDNF are abnormally regulated in the central nervous system. However, when we examined the distribution in the brain of the NGF and BDNF signal molecules using immunohistochemistry on the basis of metoclopramide's classification as a neuroleptic but weak antipsychotic agent [6], we determined that the BDNF molecule decreased in the high- and low-dose groups compared to the control group. This is in agreement with studies reporting that antipsychotic drugs cause a decrease in BDNF expression in the brain.

\section{Conclusions}

In conclusion, since metoclopramide at high doses causes a decrease in DRD2 expression in pyramidal neurons in the internal pyramidal layer in the prefrontal cortex, and since it also reduces BDNF expression in the prefrontal cortex at both high and low doses, we conclude that care should be taken over the use of metoclopramide in the lactation period due to potential extrapyramidal reactions.

Conflict Interests: The authors declare no conflict of interest.

\section{References}

1. Miller, L.G.; Jankovic, J. Metoclopramide-induced movement disorders. Clinical findings with a review of the literature. Arch. Int. Med. 1989, 149, 2486-2492.

2. Kauppila, A.; Arvela, P.; Koivisto, M.; Kivinen, S.; Ylikorkala, O.; Pelkonen, O. Metoclopramide and breast feeding: Transfer into milk and the newborn. Eur. J. Clin. Pharmacol. 1983, 25, 819-823.

3. Ehrenkranz, R.A.; Ackerman, B.A. Metoclopramide effect on faltering milk production by mothers of premature infants. Pediatrics 1986, 78, 614-620.

4. Gupta, A.P.; Gupta, P.K. Metoclopramide as a lactogogue. Clin. Pediatr. 1985, 24, $269-272$.

5. Stanley, M.; Lautin, A.; Rotrosen, J.; Gershon, S.; Kleinberg, D. Metoclopramide: Antipsychotic efficacy of a drug lacking potency in receptor models. Psychopharmacology 1980, 71, 219-225.

6. Nakra, B.R.; Bond, A.J.; Lader, M.H. Comparative psychotropic effects of metoclopramide and prochlorperazine in normal subjects. J. Clin. Pharmacol. 1975, 15, 449-454.

7. Vallone, D.; Picetti, R.; Borrelli, E. Structure and function of dopamine receptors. Neurosci. Biobehav. Rev. 2000, 24, 125-132.

(C) 2018 by the authors. Licensee MDPI, Basel, Switzerland. This article is an open access article distributed under the terms and conditions of the Creative Commons Attribution (CC BY) license (http://creativecommons.org/licenses/by/4.0/). 